\title{
Seroprevalance of Dengue and Chikungunya Infection and their Seasonal Trends
}

\author{
C. Pooja (D), Mita D. Wadekar* (D), S. Jayashree ${ }^{(D)}$ and J.V. Sathish \\ Department of Microbiology, Chamarajanagar Institute of Medical Sciences, Chamarajanagar - 571 313, \\ Karnataka, India.
}

\begin{abstract}
Dengue and Chikungunya are mosquito-borne arboviral infections with Aedes aegypti being the common vector for both. Manifestations of dengue fever closely resemble with the manifestations of Chikungunya. Although Chikungunya is a self-limiting disease, but dengue causes serious complications like Dengue Hemorrhagic Fever and Dengue Shock Syndrome. Detection of IgM antibody in Dengue and Chikungunya infection helps in early diagnosis and treatment. To study the Prevalence and Seasonal Trends of Dengue and Chikungunya infection. Two years retrospective study was conducted from January 2017 to December 2018. Serum samples from patients who were clinically suspected to have Dengue and Chikungunya were included. Samples were subjected to IgM antibody capture ELISA kits produced by NIV (Arbovirus Diagnostic NIV, Pune, India). The tests were done according to manufacturer's instruction. In 2017, Of 1664 samples tested for Dengue, 158(09.49\%) were positive and of 1439 samples tested for Chikungunya, 147(10.21\%) were positive. In 2018, of 737 samples tested for Dengue, 48 (6.51\%) were positive and of 711 samples tested for Chikungunya, 90 (12.6\%) were positive. Maximum numbers of Dengue and Chikungunya cases were seen in the age group of 21 to 30 years and 11-20 years respectively. Males were affected higher than females. Maximum seropositives were detected during Monsoon and Pre-monsoon season.The serological tests (ELISA) clearly establish the etiology and also help in initiating appropriate treatment and preventive measures in community.

Keywords: Dengue, Chikungunya, IgM antibody capture ELISA
\end{abstract}

\begin{abstract}
*Correspondence: drmdw20@gmail.com
(Received: April 17, 2020; accepted: June 22, 2020)

Citation: Pooja C, Wadekar MD, Jayashree S, Sathish JV. Seroprevalance of Dengue and Chikungunya Infection and their Seasonal Trends. J Pure Appl Microbiol. 2020;14(2):1323-1328. doi: 10.22207/JPAM.14.2.28

(C) The Author(s) 2020. Open Access. This article is distributed under the terms of the Creative Commons Attribution 4.0 International License which permits unrestricted use, sharing, distribution, and reproduction in any medium, provided you give appropriate credit to the original author(s) and the source, provide a link to the Creative Commons license, and indicate if changes were made.
\end{abstract}




\section{INTRODUCTION}

Dengue and Chikungunya are mosquitoborne, fast emerging pandemic-prone viral disease in many parts of the world. It mainly affects in urban, suburbans and the countryside and also affects in tropical and subtropical countries ${ }^{1}$. In the Philippines and Thailand in 1950s dengue was first identified and chikungunya was first recognized in the year 1952 in southern Tanzania during an outbreak. The dengue virus belongs to the genus Flavivirus, family Flaviviridae and has four serotypes (DEN-1, DEN-2, DEN-3 and DEN4). Chikungunya is an RNA virus that belongs to the alphavirus genus of the family Togaviridae ${ }^{2,3}$. These infections are transmitted by bite of various species of female mosquitoes. Aedes aegypti is the principle vector followed by Aedes albopictus. Hence, the infections are usually seen in same geographical areas ${ }^{4}$. These mosquitoes bite during the day time (early morning and late afternoon). The symptoms of both the infections closely resembles which includes Fever, Severe headache, Nausea, Vomiting, Muscle and joint pains, Pain behind the eyes, Rash and Swollen glands. Although Chikungunya is a self-limiting disease, but dengue causes serious complications like Dengue Hemorrhagic Fever (DHF) and Dengue Shock Syndrome (DSS) which mainly affects children less than 12 years and most commonly with serotype $1^{5}$.

An unusual rainfall pattern (heavy rains, deficient rains and burst in rainfall) is reflecting climate change worldwide. Few studies showed that some southern states of India are facing variations in climatic change which is affecting individuals health directly or indirectly ${ }^{6,7}$. An element of climate change like increase in average temperature supports the breeding and spread of vectors (Aedes species) which results in increase in dengue and Chikungunya infection rate ${ }^{8}$. Higher incidences are the reasons for increased morbidity and mortality worldwide ${ }^{9}$. Globally, $>2.5$ billion people are at risk and 50-100 million infections per year are likely, out of which 5 lakh cases proceed to $\mathrm{DHF}^{10}$.

Environmental conditions like season, temperature, rainfall and humidity is a major factor for the distribution of the vectors in particular geographical areas ${ }^{11-13}$. Vector survives best between the temperature of $16^{\circ} \mathrm{C}$ and $30^{\circ} \mathrm{C}$ and humidity of $60-80 \%$. Water storage in the open containers surrounding the houses supports the breeding of $A$. Aegypti ${ }^{14}$. Artificial manmade breeding sites are the main reason for outbreaks at new locations. ${ }^{[15,16]}$ The priority should be early diagnosis of the infection which allows timely management of the patient, epidemiological survey and implementation of control measures.

In order to detect these cases, there are various tests available like antigen detection tests (Non- structural 1 antigen- NS1 antigen), $\operatorname{IgM}$ and IgG antibody detection, virus isolation, immunofluorescence or by identification of viral RNA by nucleic acid amplification tests (NAAT). NS1 antigen detection for Dengue and IgM, IgG detection which detects these infections are easy to do and cheap. Moreover NS1 antigen becomes detectable from day one of fever and remains positive upto 18 days and hence helps in early diagnosis and prompt treatment ${ }^{17}$. Adequate serologic and virologic tests helps in the differential diagnosis of various febrile illnesses ${ }^{18}$.

Aim of our study is to know the seroprevalance of Dengue and Chikungunya along with seasonal trends of these infections.

\section{MATERIALS AND METHODS}

The retrospective study was done at a District hospital attached to Chamarajanagar institute of medical sciences, Chamarajanagar for a period of two years i.e, from January 2017 to December 2018.

Blood samples were collected from suspected cases of Dengue and/or Chikungunya infections.

In 2017 out of 3103 samples, 1664 samples were subjected to Dengue testing and 1439 samples for Chikungunya and in 2018 of 1448 samples received, 737 samples were tested for dengue infection and 711 samples for Chikungunya. Samples were subjected to IgM antibody capture ELISA kits produced by NIV (Arbovirus Diagnostic NIV, Pune, India). Tests were carried out according to manufacturer's instruction. Data were collected from microbiology lab registers.

\section{Statistical analysis}

Analysis was done using MS Excel.

\section{Ethical considerations}

Ethical clearance was obtained from 
Institutional Ethical Clearance Committee, Chamarajanagar Institute of Medical Science, Chamarajanagara.

\section{RESULTS}

Total of 3103 samples were tested for Dengue and Chikungunya. Out of 3103 samples received, 1664 were subjected to Dengue and 1439 to Chikungunya in 2017. In 2018 of 1448 samples received 737 samples were subjected to Dengue and 711 samples to Chikungunya. Overall seroprevalence of Dengue in our study in 2017 and 2018 was $9.49 \%(158 / 1664)$ and $6.51 \%(48 / 737)$ respectively and of Chikungunya was $10.21 \%$ (147/1439) and $12.6 \%$ (90/711) respectively. [Table-1] shows seroprevalence of Dengue and Chikungunya of the year 2017 and 2018.
Males were predominantly high-flown to Dengue infections in the year 2017 and 2018 and in Chikungunya infections, in the year 2017, Males were affected higher than female. But in the year 2018, males and females were equally affected. Gender-wise distribution is shown in [Table-2].

In 2017, Adults in the age range of 21 to 30 years were commonly involved than any other age range in both infections, which is shown in [Table-3]. In 2018, maximum cases were seen in the age group of 11-20 years as compared to any other age group in both the infections, which is shown in [Table-4]

In 2017 and 2018 Dengue infection was peak in the month of September 46 (29.1\%) and April 12 (25\%) and Chikungunya infection was peak in the month of July 47 (31.9\%) and

Table 1. Seroprevalance of Dengue and Chikungunya infection

\begin{tabular}{lcccc}
\hline Samples Tested & $\begin{array}{c}\text { Dengue } \\
\text { IgM no.s (\%) -2017 }\end{array}$ & $\begin{array}{c}\text { Chikungunya } \\
\text { IgM no.s (\%) -2017 }\end{array}$ & $\begin{array}{c}\text { Dengue } \\
\text { IgM no.s (\%) -2018 }\end{array}$ & $\begin{array}{c}\text { Chikungunya } \\
\text { IgM no.s (\%) -2018 }\end{array}$ \\
\hline Positive samples & $158(9.49 \%)$ & $147(10.21 \%)$ & $48(6.51 \%)$ & $90(12.6 \%)$ \\
Negative samples & $1506(90.50 \%)$ & $1292(89.78 \%)$ & $689(93.4 \%)$ & $621(87.3 \%)$ \\
Total & $1664(100 \%)$ & $1439(100 \%)$ & $737(100 \%)$ & $711(100 \%)$ \\
\hline
\end{tabular}

Table 2. Gender distribution of Dengue and Chikungunya infection

\begin{tabular}{lcccc}
\hline Gender & $\begin{array}{c}\text { Dengue } \\
\text { no.s (\%)-2017 }\end{array}$ & $\begin{array}{c}\text { Chikungunya } \\
\text { no.s }(\%)-2017\end{array}$ & $\begin{array}{c}\text { Dengue } \\
\text { no.s }(\%)-2018\end{array}$ & $\begin{array}{c}\text { Chikungunya } \\
\text { no.s (\%) - 2018 }\end{array}$ \\
\hline Male & $88(55.6 \%)$ & $80(54.42 \%)$ & $25(52 \%)$ & $45(50 \%)$ \\
Female & $70(44.3 \%)$ & $67(45.57 \%)$ & $23(48 \%)$ & $45(50 \%)$ \\
Total & $158(100 \%)$ & $147(100 \%)$ & $48(100 \%)$ & $90(100 \%)$ \\
\hline
\end{tabular}

Table 3. Age-wise distribution of Dengue and Chikungunya infection 2017

\begin{tabular}{lllll}
\hline $\begin{array}{l}\text { Age group } \\
\text { (years) }\end{array}$ & $\begin{array}{l}\text { Dengue } \\
\text { no.s (\%) }\end{array}$ & Positives & $\begin{array}{l}\text { Chikungunya } \\
\text { no.s }(\%)\end{array}$ & Positives \\
\hline $0-10$ & $191(11.4 \%)$ & $13(8.2 \%)$ & $198(13.7 \%)$ & $22(14.96 \%)$ \\
$11-20$ & $385(23.1 \%)$ & $14(8.8 \%)$ & $328(22.7 \%)$ & $11(7.48 \%)$ \\
$21-30$ & $421(25.3 \%)$ & $75(47.4 \%)$ & $370(25.7 \%)$ & $53(36.05 \%)$ \\
$31-40$ & $315(18.9 \%)$ & $27(17 \%)$ & $255(17.7 \%)$ & $32(21.7 \%)$ \\
$41-50$ & $167(10 \%)$ & $10(6.3 \%)$ & $147(10.2 \%)$ & $09(6.12 \%)$ \\
$51-60$ & $124(7.4 \%)$ & $07(4.4 \%)$ & $83(5.7 \%)$ & $05(3.40 \%)$ \\
$61-70$ & $43(2.5 \%)$ & $05(3.1 \%)$ & $38(2.6 \%)$ & $08(5.44 \%)$ \\
$>71$ yrs & $18(01 \%)$ & $07(4.4 \%)$ & $20(1.3 \%)$ & $07(4.76 \%)$ \\
Total & $1664(100 \%)$ & $158(9.49 \%)$ & $1439(100 \%)$ & $147(10.21 \%)$ \\
\hline Journal of Pure and Applied Microbiology & & &
\end{tabular}


Table 4. Age-wise distribution of Dengue and Chikungunya infection 2018

\begin{tabular}{lllll}
\hline $\begin{array}{l}\text { Age group } \\
\text { (years) }\end{array}$ & $\begin{array}{l}\text { Dengue } \\
\text { Samples tested } \\
\text { no.s (\%) }\end{array}$ & $\begin{array}{l}\text { Positives } \\
\text { no.s }(\%)\end{array}$ & $\begin{array}{l}\text { Chikungunya } \\
\text { Samples tested } \\
\text { no.s }(\%)\end{array}$ & $\begin{array}{l}\text { Positives } \\
\text { no.s }(\%)\end{array}$ \\
\hline $0-10$ & $85(11.5 \%)$ & $04(8.3 \%)$ & $72(10.1 \%)$ & $10(11.1 \%)$ \\
$11-20$ & $164(22.2 \%)$ & $13(27 \%)$ & $152(21.3 \%)$ & $24(26.6 \%)$ \\
$21-30$ & $187(25.3 \%)$ & $11(22.9 \%)$ & $186(26.1 \%)$ & $21(23.3 \%)$ \\
$31-40$ & $126(17 \%)$ & $11(22.9 \%)$ & $129(4.0 \%)$ & $20(22.2 \%)$ \\
$41-50$ & $93(12.6 \%)$ & $06(12.5 \%)$ & $94(13.2 \%)$ & $08(8.8 \%)$ \\
$51-60$ & $50(6.7 \%)$ & $00(0 \%)$ & $48(6.7 \%)$ & $04(4.4 \%)$ \\
$61-70$ & $18(2.4 \%)$ & $00(0 \%)$ & $17(2.3 \%)$ & $01(1.1 \%)$ \\
$>71$ yrs & $14(1.89 \%)$ & $03(6.25 \%)$ & $13(1.82 \%)$ & $02(2.2 \%)$ \\
Total & $737(100 \%)$ & $48(6.51 \%)$ & $711(100 \%)$ & $90(12.6 \%)$ \\
\hline
\end{tabular}

Table 5. Month wise distribution of Dengue and Chikungunya infection in 2017 and 2018

\begin{tabular}{llllll}
\hline \multirow{2}{*}{ Month } & \multicolumn{2}{c}{ Dengue IgM } & & \multicolumn{2}{c}{ Chikungunya IgM } \\
\cline { 2 - 3 } \cline { 5 - 6 } & 2017 & & 2018 & & 2017 \\
& No.(\%) & No.(\%) & & No.(\%) & No.(\%) \\
\hline January & 00 & $03(6.2)$ & & 00 & $03(3.3 \%)$ \\
February & 00 & $02(4.1 \%)$ & & 00 & $07(7.7 \%)$ \\
March & 00 & $04(8.3 \%)$ & & 00 & $03(3.3 \%)$ \\
April & 00 & $12(25 \%)$ & & 00 & $31(34.4 \%)$ \\
May & $17(10.7 \%)$ & $02(4.1 \%)$ & & $05(3.40 \%)$ & $04(4.4 \%)$ \\
June & $09(5.6 \%)$ & $10(20.8 \%)$ & & $36(24.4 \%)$ & $16(17.7 \%)$ \\
July & $26(16.4 \%)$ & $05(10.41 \%)$ & $47(31.9 \%)$ & $13(14.4 \%)$ \\
August & $34(21.5 \%)$ & $05(10.41 \%)$ & $23(15.6 \%)$ & $02(2.2 \%)$ \\
September & $46(29.1 \%)$ & $02(4.1 \%)$ & & $25(17 \%)$ & $05(5.5 \%)$ \\
October & $07(4.4 \%)$ & $00(0 \%)$ & & $04(2.7 \%)$ & $01(1.1 \%)$ \\
November & $12(7.5 \%)$ & $01(2 \%)$ & & $07(4.7 \%)$ & $01(1.1 \%)$ \\
December & $07(4.4 \%)$ & $02(4.1 \%)$ & & $00(0 \%)$ & $04(4.4 \%)$ \\
Total & $158(100 \%)$ & $48(100 \%)$ & $147(100 \%)$ & $90(100 \%)$ \\
\hline
\end{tabular}

April 31 (34.4\%) which is shown in the [Table- 5] respectively.

\section{DISCUSSION}

Aedes aegypti mosquito pass on both the Dengue and the Chikungunya virus. Clinical features are shared by both infections during the acute phase like fever, joint and bone pain, nausea, vomiting, headache, and fatigue. Clinical features of Dengue and Chikungunya are similar but their outcome differs. In 2013, the fifth serotype (DEN5) was discovered from Bangkok. Chikungunya is mostly nonfatal while dengue can cause severe illness including death ${ }^{5}$.

In India the disease is prevalent in almost all states and union territories. Between 2010-
17, >6 lakh cases with >1560 deaths have been reported from West Bengal, Tamilnadu, Punjab, Kerala, Delhi, Karnataka, and Maharashtra. Of $1,57,220$ cases reported in 2017, maximum were reported from Tamilnadu followed by West Bengal isolating all four Dengue serotypes. Serotype prevalence varies between seasons and places, but DEN-1 and DEN-2 are widespread. DEN-5 has not been reported yet.

In our study Dengue prevalence was (9.49\%) and (6.51\%) (Table-1) which correlates with the study of Shashi sharma sudhan et.al. who reported the prevalence of $21 / 229$ (9.1\%) of Dengue ${ }^{2}$ and Chikungunya prevalence was (10.21\%) and (12.6\%) (Table-1) which correlates with the study of Anju Dinkara et.al. who reported 
prevalence of $23(12.37 \%)$ cases for chikungunya ${ }^{19}$. In our study Dengue positivity was predominantly between the age range of 21-30 years i.e. (47.4\%) in 2017 and $11-20$ years i.e. $(27 \%)$ in 2018 . Our study correlates with the study of Arvind Neralwar et,al. who reported the majority of the dengue illness between the age group of 21 to 30 years $(41.44 \%)^{17}$. Chikungunya positivity was also predominant in the age range of $21-30$ years i.e. (36.05\%) in 2017 and 11-20 years i.e. (26.6\%) in 2018 which correlates with the study of Modi K.P. et al. who reported the majority of the dengue illness between the age group of 21 to 30 years $279(35.09 \%)^{5}$.

In our findings we can observe the age shift in the dengue and chikungunya patients from being in adults (21-30 years) to children and young adolescents (11-20 years). Singapore, Indonesia, and Thailand studies suggested that individuals developed multitypic immunity following exposure to different serotypes. Over a period of time as co-circulation of different serotypes increases, individuals remain lesser susceptibility to infection. Hence, younger age group individuals become completely susceptible. Therefore, individuals immune to specific serotype are more likely to be from younger age groups ${ }^{20}$.

In our study Dengue positivity was predominantly in the males i.e, (55.6\%)-2017, (52\%)-2018 which correlates with the study of Arvind Neralwar which has shown a male preponderance of $63.19 \%$ in the clinically suspected dengue patients ${ }^{17}$. and Chikungunya positivity was predominantly in the males i.e, (54.42\%) in 2017 which correlates with the study of Usha Kalawat et.al, which has shown male preponderance of 96 (56\%) in the clinically suspected Chikungunya patients ${ }^{21}$. But in 2018, Chikungunya was equally affected in both male and female. i.e. (50\%) in each. The reason for the excess of reported male dengue and chikungunya cases is observed in this study can be due to day time working hours and their exposure to dengue and Chikungunya carrying mosquitoes.

In our study, the suspected Dengue infection was peak in the monsoon season i.e. in the month of September, $(29.1 \%)$ in 2017 and June $(20.8 \%)$ in 2018 . Similarly, our study correlates with the study of Sujet Lertanekawattana et.al, in which dengue cases decreased after the last week of July; however, reached its highest point in mid-August and cases continued until the end of September ${ }^{22}$. Chikungunya infection was peak in monsoon and pre-monsoon i.e. in the month of July, (31.9\%) in 2017 and April (34.4\%) in 2018. Our study correlates with the study of Akanksha Tomar et al. Here, a seasonal peak of Chikugunya infection was seen in the months of July to September, while showing major decline in other seasonal months ${ }^{23}$. Aedes aegypti is sensitive to climatic changes like temperature and moisture. Increased temperature and wet season favours its survival whereas dry and cool seasons does not ${ }^{21}$.

Clinically suspected cases should be tested for both Dengue and chikungunya infections especially in endemic areas, as the signs and symptoms of these infections overlap. As a result of urbanization in India, there is increase in the incidence of dengue infection. Hence continuous surveillance of these viruses will help for its identification and characterization. This information will also help to control outbreak by implementing effective control measures ${ }^{5}$.

\section{CONCLUSION}

The Dengue and Chikungunya are the most common arboviral infections. These infections continue to co-exist in our country and there is substantial overlap in clinical presentation between these infections. So, simple clinical criteria along with lab investigations will help for appropriate management of these infections. The serological results (IgM antibody capture ELISA) clearly establish the etiology. The findings of age shift may be of help to the epidemiologists, paediatricians, and prevention of Dengue and Chikungunya infections and control measure targeting the young population.

\section{ACKNOWLEDGMENTS}

The author extends gratitude to all the colleagues who helped in the study.

\section{CONFLICT OF INTEREST}

The authors declare that there is no conflict of interest. 


\section{AUTHORS' CONTRIBUTION}

PC drafted the manuscript, compiled the information and designed the tables. MDW and JS gathered information. SJV supervised and reviewed the manuscript and tables.

\section{FUNDING}

None.

\section{ETHICS STATEMENT}

Ethical clearance was obtained from Institutional Ethical Clearance Committee, Chamarajanagar Institute of Medical Science, Chamarajanagara, India.

\section{DATA AVAILABILITY}

All datasets generated or analyzed during this study are included in the manuscript.

\section{REFERENCES}

1. Bhooshan S Gandhi et al. Dengue and Chikungunya coinfection associated with more severe clinical disease than mono-infection. Pune, India. International Journal of Healthcare and Biomedical Research. 2015;03(03):117-123.

2. Shashi sharma sudhan et al. Serosurveillance of Dengue, Chikungunya and Zika in Jammu, a Sub-Himalayan Region of India. J Clin Diagn Res. 2017;11(11):DC05-DC08.

3. Synthia Selvakumari. A Study on Detection of Prevalence of Dengue, Chikungunya, Leptospirosis and their Coinfection in Acute Febrile Patients, Madras, India. University Journal of Pre and Para Clinical Sciences. 2018;4(1):2455-2879

4. Farah Deeba, Co-infection with Dengue and Chikungunya Viruses, Chapter 6-Current Topics in Chikungunya. Published by World's largest Science, Technology \& Medicine Open Access book publisher.

5. Modi KP et al. Sero-Prevalence of Dengue and Chikungunya, their Co-Infection and Seasonal Trends of These Infections at a Tertiary Care Hospital, Ahmedabad, Gujarat. Int I Microbiol Res. 2017;9(1):819-822.

6. WHO, Climate change and human health: risk and responses. World Health Organization, Geneva, 2003; http://www.who.int/ globalchange/publications/ climchange.pdf

7. Singh PK, Dhiman RC. Climate change and human health: Indian context. Journal of Vector Borne Diseases. 2012;49(2):55-60.

8. Mishra R, Kumar P. A study on outbreak of dengue from Bihar, India establishing new foci, attributable to climatic changes. Journal of Public Health Epidemiology. 2011;3(11):489-502.

9. Gubler DJ, Dengue and Dengue Haemorrhagic Fever,
Seminars in Pediatric Infectious Diseases, 1997;8(1):39. https://doi.org/10.1016/S1045-1870(97)80003-9

10. Guzman MG, et al. Dengue: a continuing global threat. Nature Rev Microbiol. 2010;S7-S16. https://doi. org/10.1038/nrmicro2460

11. Johansson MA, Dominici F, Glass GE. Local and global effects of climate on dengue transmission in Puerto Rico. PLoS Neglected Trop Dis. 2009;3(2):e382. https:// doi.org/10.1371/journal.pntd.0000382

12. Chinery WA. A survey of mosquito breeding in Accra, Ghana during a two-year period (Sept. 1964-Aug. 1996) of larval mosquito control iii. The breeding of Aedes aegypti, Linnaeus, in Accra. Ghana Med J. 1970;9:197-200.

13. Surtees $\mathrm{G}$. The distribution, density and seasonal prevalence of Aedes aegypti in West Africa. Bull WHO. 1967;36(4):539-540.

14. NVBDCP, Guidelines for clinical management of dengue fever, dengue haemorrhagic fever and dengue shock syndrome, 2008.

15. Ilkal MA, et al. Entomological investigations during outbreaks of dengue fever in certain villages in Maharashtra state. Indian J. Med. Res. 1991;93:174178.

16. Mehendale SM, Risbud AR, Rao JA, Banerjee K. Outbreak of dengue fever in rural areas of Parbhani district of Maharashtra (India). Indian J. Med. Res. 1991;93:6-11.

17. Arvind Neralwar, et al. Detection of NS1 antigen, IgM antibody for the diagnosis of dengue infection in patients with acute febrile illness. International Journal of Research in Medical Sciences. 2015;3(10):2826-2830. https://doi.org/10.18203/2320-6012.ijrms20150833

18. Isabel Rodriguez-Barraquer. The Hidden Burden of Dengue and Chikungunya in Chennai, India. PLOS Neglected Tropical Diseases | DOI:10.1371/journal. pntd.0003906 July 16, 2015

19. Anju Dinkara. Hidden burden of chikungunya in North India; A prospective study in a tertiary care centre. $J$ Infect Pub Health. 2018;11(4):586-591. https://doi. org/10.1016/j.jiph.2017.09.008

20. Yukti Sharma, Mandeep Kaur, Sompal Singh, Leela Pant, Madhur Kudesia, Sanjay Jain. Seroprevalence and Trend of Dengue Cases Admitted to a Government Hospital, Delhi -5-Year Study (2006-2010): A Look into the Age Shift. Int J Prev Med. 2012;3(8):537-543.

21. Usha Kalawat et al. Prevalence of dengue and chickungunya fever and their co-infection. Indian Journal of Pathology and Microbiology. 2011;54(4): ip: 157.49.37.138.

22. Sujet Lertanekawattana. Prevalence and characteristics of Dengue and Chikungunya infections among acute febrile patients in Nong Khai province, Thailand

23. Ms. Akanksha tomar. prevalence of Chikungunya viral infection in a tertiary care hospital, navi mumbai Maharashtra.

24. Haque HFa. Dengue-Chikungunya Co-Infection: A Case Report from 2017-Chikungunya Outbreak in Dhaka, Bangladesh. Birdem Medical Journal. 2018;8(1). https://doi.org/10.3329/birdem.v8i1.35044 